\title{
Correlation of an ex Vivo Model with Clinical Application of an Epigenetic Modifier, Inhibiting Tumor Growth and Metastasis, in Resistant Cholangiocarcinoma-A Case Study
}

\author{
M. A. Nezami' ${ }^{1}$, Aron Gould Simon², Geoffrey Bartholomeusz ${ }^{3}$ \\ ${ }^{1}$ Pacific Medical Center of Hope Inc., Fresno, CA, USA \\ ${ }^{2}$ Valley Metabolic Imaging (VMI), Fresno, CA, USA \\ ${ }^{3}$ Si RNA Department, Experimental Therapeutics, MD Anderson Cancer Center, Houston, TX, USA \\ Email: amnezami@yahoo.com
}

Received 21 December 2015; accepted 25 January 2016; published 28 January 2016

Copyright (C 2016 by authors and Scientific Research Publishing Inc.

This work is licensed under the Creative Commons Attribution International License (CC BY). http://creativecommons.org/licenses/by/4.0/

(c) (7) Open Access

\section{Abstract}

Bile duct cancer is a rare form of cancer, with approximately 2000 new cases diagnosed in the United States each year. The prognosis of this disease is very grave, especially in the form of intrahepatic (IHCC), as there is no person with stage four who lives for 5 years, and the average prognosis is less than a year, a majority of patients die in less than $\mathbf{6}$ months despite all therapies. It is suggested that one of the key elements in the disease progression is the intratumoral hypoxia inducible factor one alfa (HIF-1a) as a regulator of malignant behavior and recently described as a new prognostic indicator of IHCC. $(9,10) \mathrm{HIF}$ is a key regulator under the microenvironmental (terrain) influence, and therefore studies of the cell lines in an in vitro environment where there is no hypoxia, usually fail to translate to a clinical outcome in vivo, unless the cells are transfected by fulllength HIF-1alpha (fL HIF-1alpha) and dominant-negative HIF-1alpha (dn HIF-1alpha). To overcome this barrier, an ex vivo model is designed at MD Anderson experimental therapeutics where the patient tumor sample is transferred to the mice and treated with drugs, where the tumor can cross talk with the actual terrain and mimic the human stroma where the HIF can be triggered. Results show significant tumor necrosis on the intrahepatic cholangiocacinoma, only after 5 days of exposure to an experimental compound that is known to suppress hypoxia-induced accumulation of hypoxia-inducible factor- $1 \alpha$ (HIF-1 $\alpha$ ) through inhibiting protein synthesis. $(11,12)$ Further this is explored in the same actual patient with terminal diagnosis, and proves itself with promising initial response. Here, we review this method and the clinical perspectives, and suggest this method to be studied in larger trials. 


\section{Keywords}

\section{Cholangiocarcinoma, Epigenetic Therapies}

\section{Background}

Studies recently have shown a great influence in cancer progression and metastasis induced by terrain. While all the prior focus of efforts is on inhibiting angiogenesis, the role of terrain in promoting invasion is not revealed, until recently when it is shown that by inhibiting angiogenesis, tumor invasion increases due to changes in the microenvironment [1]. This abstract describes a method of interference with this effect (microenvironemntal seduction), to reverse invasion and further metastasis.

One of the major focuses of inhibiting angiogenesis begins with the development of drugs to block VEGF (vascular endothelial growth factor), which theoretically will cause tumor regression by blocking its source of nutrients and oxygen. We have learned that this strategy fails due to many resistance mechanisms, such as the induction of feedback loops. Through the recent work of researchers at UCSF, we learn that the engagement of bone marrow derived cells (BMDC) through hypoxia induced factor (HIF) alpha-1, secondary to SDF alpha-1, contributes to VEGF binding to VEGF receptor 2 [2] [3]. Therefore, we can theoretically conclude that blocking VEGF itself, although lowers the VEGF (initially), can increase the binding of VEGF to VEGF receptor 2 through indirect activation of HIF and stimulation of BMDC (The bone marrow derived cells are positive for CD 45 and MMP 9, which interestingly are able to cleave the VEGF allowing it to bind to its receptor) [2] [3].

This understanding has also yielded to understanding of the role of bone marrow driven cells in forming the microenvironment itself. Terrain, as the name suggests that it provides the protection for the tumor, as the cancer cells intelligently recruit the bone marrow cells into their microenvironment. Hypoxia also stimulates the terrain to recruit other promoting cells. The terrain recruits microglial cells in the case of brain cancer, stimulated by the tumor, through mechanisms suggested earlier, such as Transforming Growth Factor-1 and Interleukin-34, which they are hypoxia driven [4]. The microglial cells then produce HGF, CTGF, IL-4 and 6, which are hormonally dependent [5]. This endocrine machine is activated through PI3K pathway gamma in these cells, and stimulates tumor cells mesenchymal features and induces invasion [6]. Laboratory findings have confirmed that blocking the microglial PI3K is able to inhibit this seduction and recruitment to microenvironment [7]. Blocking the HIF can potentially inhibit the cMET through PTP1B, and tumor cell invasion [8]-[11]. Technically, such a strategy will be both clinically relevant and prognostically significant in the clinical setting. We have shown in both preclinical as well as clinical models, that using multi-targeted epigenetic therapy (MTET), a protocol which targets HIF, we are able to reduce the serum VEGF, as well as tumor migration and invasion in 3-dimensional culture media. These results confirm that we are able to reduce the tumor angiogenesis, and at the same time, tumor invasion is decreased, by modifying the microenvironment seduction pattern. We conclude that tumor mesenchymal features and the epithelial-mesenchymal transition (EMT) are somehow dependent on the activation of the HIF, and further seduction of the microenvironment, explaining the clinical findings in our case study.

\section{Case Study}

82 year old female (SZ) with history of stage IV intrahepatic cholangiocarcinoma, diagnosed in 2012 after multiple episodes of jaundice/cholangitis. At her initial evaluation she was status post stereotactic body radiation therapy at MD Anderson Cancer Center, which resulted in shrinkage of the mass, however the tumor rapidly progressed with multiple pulmonary metastasis documented in her CT scan on 6/2/2014.

She had a biopsy of a liver lesion done on 6/4/14, as well as circulatory tumor DNA testing, which confirmed KRAS, BRAF and SMAD4 mutations. She was referred to our clinic, by M D Anderson oncology, after initial ex vivo model laboratory findings done at MD Anderson confirmed positive predicted response to the therapies implemented at our clinic under MTET (multi-targeted epigenetic therapies) protocol. There was significant tumor necrosis, verified in matter of 3 - 5 days after exposure to different doses of the drug (Please see the below Figure 1).

At this time, she had a terminal diagnosis and further refused any chemotherapies, considering the toxicity 
06-04-2014-Received fresh liver core biopsy (very small piece)

06-04-2014-06-30-2014-processed tumor cells and grew ex-vivo tumor in laboratory

07-01-2014-divided ex-vivo tumor into smaller pieces and placed each piece into individual well of a 96 well plate

07-11-2014-Treated the divided ex-vivo tumor with varying concentrations of (Compound X/Quercetin)

07-14-2014-Noticed phenotypic change of ex-vivo tumor tissue on day 3 of treatment

07-16-2014-Images tsken of treated ex-vivo tumor (day 5 of treatment)

control-untreated media only

DMSO-concentration of DMSO equal to that in the $200 \mu \mathrm{M}$ sample of Compound X/Quercetin to rule out any effect of DMSO

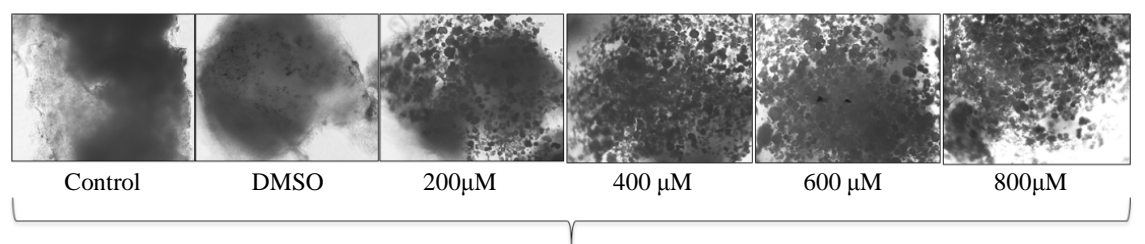

Concentration of Compound X (Epigenetic therapy/Quercetin)

Figure 1. Ex vivo sample of cholangiocarcinoma treated with compound X/quercetin.

and her age. She fell into high risk patients with following criteria, studied in multivariate analysis: metastatic disease, intrahepatic primary site, the presence of liver metastases, poor Eastern Cooperative Oncology Group (ECOG) performance status and elevated level of serum alkaline phosphatase all, significant negative predictors of overall survival. It is of note that all conventionally used chemotherapy regimens have failed to show significant improved survival, compared to palliative care or hospice, in this group of patients.

Secondary to her initial tumor response, she was referred to the clinic. Initially, upon her arrival she was having pain and general weakness. At our clinic, she underwent an extensive work up, which included a staging PET scan, and evaluation of prognostic surrogate markers of survival consisting of growth factors, and purified disseminated tumor cells DNA. These test confirmed presence of circulatory tumor cells with increased cMYC activity in serum, increased serum HER2 levels, and elevated tumor markers (CEA, CA 125, CA 15.3 and CA 19.9). She was found to have positive CTC in blood as well.

Her staging PET scan showed active masses in liver, which were "substantially" increased size compared to her prior CT scan dated June 2014 (Please see below).

The largest mass was reported in left lobe of the liver about $8 \mathrm{~cm}$ in size, increased from $6 \mathrm{~cm}$ (about 200\% increase in size) compared to prior scan on 6/2/14, with metabolic activity of 9 , and pulmonary lesions too numerous to count.

She was immediately started on MTET protocol, which consisted of IV administration of polyphenol (Quercetin), which she received on daily basis, per protocol [12]. Immediately she reported improved quality of life (by ECOG) and reduction of her pain level and increased general stamina. All her labs were repeated on 8/8/14, after 10 days of therapy.

The following laboratory results were obtained:

There was a reduction of the tumor markers (CA 15.3, CA 19.9, CA 125, and CEA) from expected growth velocity, as her tumor markers had doubled over the course of one month, prior to the therapy. Her CEA dropped from 1343 down to 1221 (measured on 8/8/14). The circulatory tumor cell assay showed complete response to interim therapy, and no evidence of circulatory tumor cells post-therapy were seen (Measured on 8/12/14). Her liver enzymes also improved.

Alkaline Phosphatase (ALK-P) improved from 265 to 238. CA 19.9 dropped from 113013 to 99186, and CA 15.3 dropped from 28.3 to 26 (This response although was not sustained, it was the first time in her history of disease that we were able to stabilize and possibly regress the disease, by reduced velocity of her tumor growth). Serum HER2 level dropped from 15.5 to 11.8 (normalized) (See Figure 2 below). It is of note that she also had a history of non Hodgkin's lymphoma, (treated in 2011, status post R-CHOP therapy), and her Interleukin 2 receptor was checked prior to therapy and monitored during the treatments. Her IL-2 receptor normalized posttherapy, initially tested at 725 and dropped to less than 31.2 (undetectable).

The following are the reported by imaging: 
The pulmonary lesions growth was inhibited and somewhat stabilized with therapy, both in size and metabolic activity. This was a positive response compared to the prior extensive and rapid progression of disease, between June 2nd 2014 and July 1st 2014 (PRE therapy). The trend of the growth in liver lesions also decreased post therapy (See below Table 1, Table 2).

Lesion comparison on patient SZ.

Study 1 = CT abdomen pelvis of 6/2/14.

Study $2=$ PET/CT of 7/30/14.

Study $3=$ PET/CT of 8/29/14.

Further, the patient decided to withhold the treatments due to financial and family circumstances, and proceed with other experimental therapies at home, which she was advised against. Her response was not durable in her lab findings due to her inconsistent follow up and non-compliance, nevertheless her rapid clinical response suggested by stabilization of her metastatic disease and response in her markers are suggestive of a strong correlation with ex vivo laboratory findings. This further warrants clinical trials in a larger sample size for patients with advanced metastatic disease, implementing this model to apply in clinical settings.

\section{Serum HER-2}

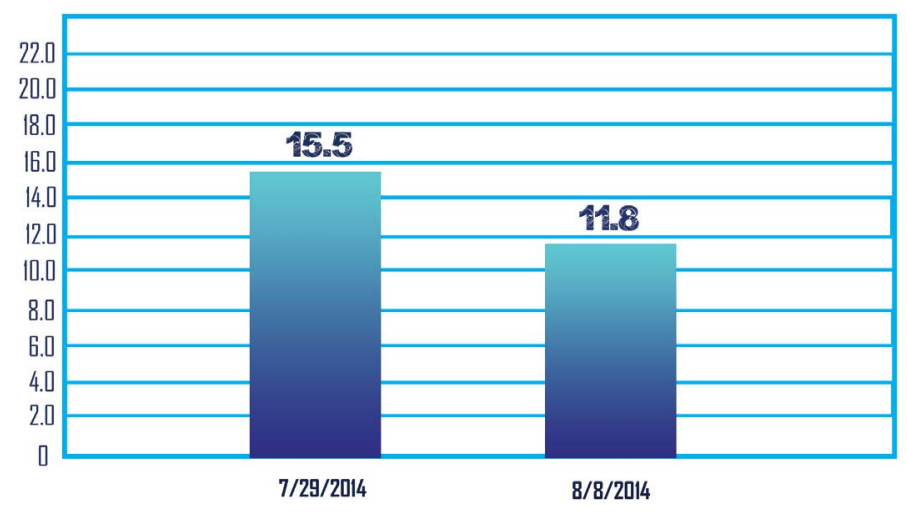

Figure 2. Patient SZ's pre and post serum HER-2 levels.

Table 1. PET/CT-representative lung lesions.

\begin{tabular}{|c|c|c|}
\hline Representative lung lesions & PET/CT of 7/30/14 & PET/CT of 8/29/14 \\
\hline Lung nodule 1 & $6 \times 6 \mathrm{~mm}, \mathrm{SUV} 1.0$ & $6 \times 6 \mathrm{~mm}, \mathrm{SUV} 1.5$ \\
\hline Lung nodule 2 & $6 \times 6 \mathrm{~mm}, \mathrm{SUV} 1.1$ & $6 \times 7 \mathrm{~mm}$, SUV 2.5 \\
\hline Lung nodule 3 & $5 \times 5 \mathrm{~mm}$, SUV 0.9 & $5 \times 5 \mathrm{~mm}$, SUV 1.0 \\
\hline Lung nodule 4 & $5 \times 6 \mathrm{~mm}$, SUV 1.2 & $5 \times 6 \mathrm{~mm}$, SUV 2.7 \\
\hline Lung nodule 5 & $9 \times 5 \mathrm{~mm}$, SUV 1.3 & $8 \times 6 \mathrm{~mm}$, SUV 1.1 \\
\hline Lung nodule 6 & $4 \times 3 \mathrm{~mm}$, SUV 1.0 & $4 \times 3 \mathrm{~mm}$, SUV 1.1 \\
\hline Lung nodule 7 & $4 \times 3 \mathrm{~mm}$, SUV 0.9 & $4 \times 3 \mathrm{~mm}$, SUV 0.8 \\
\hline
\end{tabular}

Table 2. PET/CT-representative liver lesions.

\begin{tabular}{cccc}
\hline Representative liver lesions & CT abdomen pelvis of 6/2/14 & PET/CT of 7/30/14 & PET/CT of 8/29/14 \\
\hline Dominant mass & $7.7 \times 3.7 \mathrm{~cm}$ & $8.2 \times 4 \mathrm{~cm}$, SUV 9.9 & $8.5 \times 4.3 \mathrm{~cm}$, SUV 12.2 \\
Lesion 2 & $1.3 \times 1.1 \mathrm{~cm}$ & $1.5 \times 1.2 \mathrm{~cm}$, SUV 4.8 & $1.7 \times 1.6 \mathrm{~cm}$, SUV 6.4 \\
Lesion 3 & & $1.3 \times 1.1 \mathrm{~cm}$, SUV 5.3 & $1.5 \times 1.3 \mathrm{~cm}$, SUV 7.2 \\
Lesion 4 & $0.7 \times 0.5 \mathrm{~cm}$, SUV 1.8 & $0.8 \times 0.7 \mathrm{~cm}$, SUV 1.9 \\
Lesion 5 & & $0.8 \times 0.6 \mathrm{~cm}$, SUV 4.4 & $1.0 \times 1.0 \mathrm{~cm}$, SUV 10.2 \\
\hline
\end{tabular}




\section{Conclusion}

Microenvironmental influences in cancer experimental therapeutics are a major barrier in translating results from in vitro to in vivo models, and a big cause of new drug failures in human. This case represents a promising method of treating cholangiocarcinoma, with a novel approach, by one of the best reproducible techniques, in ex vivo model, translating same tumor cell response, from mice into human. Application of available scientific knowledge and generating further hypothesis can be possible by using such model to expand our capabilities in new drug development. Further clinical trials are warranted for using quercetin, and other polyphenols applied in multitargeted epigenetic therapy (MTET), considering the large antitumor effect size in this case.

\section{References}

[1] Quail, D. and Joyce, J. (2013) Microenvironmental Regulation of Tumor Progression and Metastasis. Nature Medicine, 19, 1423-1437. http://dx.doi.org/10.1038/nm.3394

[2] Du, R., Lu, K., Petritsch, C., Liu, P., Ganss, R., Passegué, E., et al. (2009) HIF1 $\alpha$ Induces the Recruitment of Bone Marrow-Derived Vascular Modulatory Cells to Regulate Tumor Angiogenesis and Invasion. Cancer Cell, 13, $206-220$.

[3] Chan, D., Kawahara, T., Sutphin, P., Chang, H., Chi, J. and Giaccia, A. (2009) Tumor Vasculature Is Regulated by PHD2-Mediated Angiogenesis and Bone Marrow-Derived Cell Recruitment. Cancer Cell, 15, 527-538. http://dx.doi.org/10.1016/j.ccr.2009.04.010

[4] Bhattacharya, D., Chaudhuri, S. and Chaudhuri, S. (2014) Abstract 1024: T11TS Impedes Glioma Angiogenesis by Attenuating Brain Endothelial Angiopoietin-1/Tie2 Signaling and Inducing Apoptosis of Glioma Associated Brain Endothelial Cells. Cancer Research, 74, 1024-1024. http://dx.doi.org/10.1158/1538-7445.AM2014-1024

[5] Li, W. and Graeber, M. (2012) The Molecular Profile of Microglia under the Influence of Glioma. Neuro-Oncology, 14, 958-978. http://dx.doi.org/10.1093/neuonc/nos116

[6] Larue, L. and Bellacosa, A. (2005) Epithelial-Mesenchymal Transition in Development and Cancer: Role of Phosphatidylinositol 3' Kinase/AKT Pathways. Oncogene, 24, 7443-7454. http://dx.doi.org/10.1038/sj.onc.1209091

[7] Huang, J., Ding, Y., Huang, D., Liang, A., Zeng, W., Zeng, Z., et al. (2013) Inhibition of the PI3K/AKT Pathway Reduces Tumor Necrosis Factor-Alpha Production in the Cellular Response to Wear Particles in Vitro. Artificial Organs, 37, 298-307. http://dx.doi.org/10.1111/j.1525-1594.2012.01568.x

[8] Lu, K., Chang, J., Parachoniak, C., Pandika, M., Aghi, M., Meyronet, D., et al. (2012) VEGF Inhibits Tumor Cell Invasion and Mesenchymal Transition through a MET/VEGFR2 Complex. Cancer Cell, 22, 21-35. http://dx.doi.org/10.1016/j.ccr.2012.05.037

[9] Morine, Y., Shimada, M., Utsunomiya, T., Imura, S., Ikemoto, T., Mori, H., et al. (2011) Hypoxia Inducible Factor Expression in Intrahepatic Cholangiocarcinoma. Hepatogastroenterology, 58, 1439-1444. http://dx.doi.org/10.5754/hge11156

[10] Vanichapol, T., Leelawat, K. and Hongeng, S. (2015) Hypoxia Enhances Cholangiocarcinoma Invasion through Activation of Hepatocyte Growth Factor Receptor and the Extracellular Signal-Regulated Kinase Signaling Pathway. Molecular Medicine Reports, 12, 3265-3272. http://dx.doi.org/10.3892/mmr.2015.3865

[11] Lakka, A., Mylonis, I., Bonanou, S., Simos, G. and Tsakalof, A. (2010) Isolation of Hypoxia-Inducible Factor 1 (HIF-1) Inhibitors from Frankincense Using a Molecularly Imprinted Polymer. Investigational New Drugs, 25, 1081-1089.

[12] Lee, D. and Lee, Y. (2008) Quercetin Suppresses Hypoxia-Induced Accumulation of Hypoxia-Inducible Factor-1 $\alpha$ (HIF-1 $\alpha$ ) through Inhibiting Protein Synthesis. Journal of Cellular Biochemistry, 105, 546-553.

http://dx.doi.org/10.1002/jcb.21851 